\title{
Identification of Different Isolated Fungi from Scaly Debris of Colocasia Corm With Potential Production of Cellulase
}

\author{
Nahla Alsayed Bouqellah \\ Biology Department, Science College, Taibah University, 42317- 8599, Al Madinah \\ Al Munawwarah, Saudi Arabia.
}

\begin{abstract}
$\mathbf{T}$ HE AIM of the present work is to isolate and identify the different fungi from the debris of the brown outer scaly leaves of Colocasia corm. The isolated fungi were tested for cellulase producing activity. The species were primarily recognized on morphological bases and then identified on molecular level. The Internal Transcribed Spacer (ITS) domain of the ribosome large subunit (LSU) comprising the ITS1/5.8S/ITS2 regions ( $\geq 500 \mathrm{bp}$ ) was subjected to Sanger sequencing for the new isolates using primer pair ITS1/ITS4. BLAST analysis indicated entities of the eight isolated fungal isolates. Among the isolates, three of the species were found closely related to Fusarium solani whereas, the other five isolations were found to be closely related to F. proliferatum, F. fujikuroi, F. equiseti, Penicillium lanosum and $P$. flavigenum. Screening of the fungal species for cellulase production was performed on cellulose agar plates using clear zone technique. The results showed that out of theses isolates, the maximum zone of clearance was obtained for isolate that is closely related to P. lanosum, whereas, the minimum zone of clearance was recorded for isolates closely related to Fusarium solani. Penicillium isolate showing significant potentiality in cellulase production will be a subject for a detailed investigation.
\end{abstract}

Keywords: Plant debris, Penicillium, Fusarium, rDNA, ITS, Cellulase.

\section{Introduction}

Cellulose is a major renewable form of carbohydrate, with approximately $10^{11}$ tons produced annually. Cellulose is a linear unbranched homopolymer of glucose joined together via $\beta$ 1-4 glycosidic linkages and are vary in length and arranged in bundles or fibrils (Walsh, 2015). It is the most abundant component of plant biomass that is found in nature almost exclusively in plant cell walls, albeit cellulose is produced by few fungi (oomycetes), animals (tunicates) and bacteria. However, the plant materials are considered the ideal supply of cellulose to the environment (Lynd et al., 2002). Within the bundles, cellulose molecules can occur in crystalline or paracrystalline (amorphous) structures (Walter, 1998).

Processes to efficiently and economically convert cellulosic materials to glucose are of massive industrial significance (Walsh, 2015). However, the crucial approach in cellulose conversion is the enzymatic hydrolysis through cellulase enzyme. Cellulase catalyzes the conversion of insoluble cellulose to simple, water soluble products (Alexander, 1961). Cellulase is a complex system of enzymes, comprising endoglucanase (endo-1,4- $\beta$-D-glucanase, EC 3.2.1.4), exoglucanase (exo- 4- $\beta$-D-glucancellobiohydrolase, EC 3.2.1.91) and $\beta$-Dglucosidase ( $\beta$-D-glucoside glucanhydrolase, EC 3.2.1.21). These enzymes act synergistically to degrade cellulosic substrates in which action of the first and the second involves the recovery of short cellulose chains, while the third makes complete breakdown into glucose (Singhania et al., 2010 and Sajith et al., 2016). Previous studies indicated that the number of genes governing the production of endoglucanase enzyme is two, while eight and seven for exoglucanase and glucosidase enzymes, respectively (Aro et al., 2005 and Sukumaran et al., 2005).

Fungi and bacteria are the main natural potent agents for cellulose degradation (Lederberg, 1992). The cellulose-utilizing organisms include aerobic and anaerobic mesophilic bacteria, filamentous fungi, thermophilic and

\#Corresponding author email: nahla.b@hotmail.co.uk DOI: 10.21608/ejbo.2019.7575.1283

Edited by: Prof. Dr. Soliman A. Haroun, Faculty of Science, Kafr El-Sheikh University, Kafr El-Sheikh, Egypt. (C)2019 National Information and Documentation Center (NIDOC) 
alkaliphilic bacteria, actinomycetes and certain protozoa (Alexander, 1961). However, fungi are the main cellulase-producing microorganism that is also known for decomposing other organic matter (Lynd et al., 2002). Examples of cellulaseproducing fungi include species of the genera Fusarium and Penicillium, in addition to those of genera Basidiobolus, Aspergillus, Arthroderma, Geotrichum, Arthrographis and Alternaria (Vries \& Visser, 2001).

Cellulases have been used for years in several industries including food processing, feed preparation, wastewater treatment, textile production, paper and pulp production. Additional potential applications include the production of wine, beer and fruit juice (Philippidis, 1994). Furthermore, glucose produced from degrading cellulosic substrate by cellulase could further contribute as a substrate in fermentation or processes which could yield valuable end products such as ethanol, butanol, methane, amino acids, single-cell protein and others (Walsh, 2002).

The present work aims at isolating and identification of new fungal isolates grown on rotten peel of Colocasia corm at the morphological and molecular levels and detect their potential ability to produce cellulase enzyme.

\section{Materials and Methods}

\section{Colocasia corm}

Colocasia rotten corms were obtained from the local market of Al-Madinah Al Munawwarh. Corms were brought to the laboratory and kept at the refrigerator until further used.

\section{Isolation and morphological identification of the recovered fungi \\ Czapek Doxs agar medium (HiMedia,} Mumbai, India) was used for growing fungi. Fungi grown on dry scales of a rotten Colocasia were picked up by a sterile needle and placed on Petri dishes containing sterilized Dox's agar medium. The plates were incubated at $30^{\circ} \mathrm{C}$ for 7 days, then the fungal isolates were purified and identified morphologically up to the species level via microscopic examination (Gilman, 1950; Gomori, 1955; Ellis, 1971; Moubasher, 1993 and Seifert, 1996). The recovered isolates were named as M1-M8.

\section{Molecular identification of fungi}

DNAs of the eight isolates were extracted and sent to Macrogen Inc. (Seoul 08511, Rep. of Korea) for genus and species identification via Sanger sequencing. The analysis used for the identification of the eight fungal isolates was based on the divergence of rDNA gene region namely internal transcribed spacer or ITS (Fig. 1). Two taxon-specific primers (White et al., 1990) used were namely ITS1 (5 TCCGTAGGTGAACCTGCGG $3^{\prime}$ ) and ITS4 (5' TCCTCCGCTTATTGATATGC 3`) were used for forward and reverse sequencing of the ITS region $(>450 \mathrm{bp})$. The obtained sequencing results were submitted to the National (NCBI, https://www.ncbi.nlm.nih.gov/) and received accession numbers MH553280-MH553287 referring to isolates M1-M8, respectively. These sequences were subjected to BLAST search engine (https://blast.ncbi.nlm.nih.gov/Blast. cgi) for the identification of the fungal species with similar sequences in this rDNA region. Nucleotide sequences of the eight isolates alone as well as along with those retrieved from the gene bank were aligned in Ugene (Okonechnikov et al., 2012) using the T-Caffee algorithm (https:// www.ebi.ac.uk/, EMBL-EBI, Cambridgeshire, CB10 1SD, UK) and a phylogenetic cladogram was inferred. One of the two trees generated from Ugene program was displayed using iTOL/ interactive tree (http://itol.embl.de/index.shtml), (Letunic \& Bork, 2011) in order to describe the phylogenetic relationships among the new isolates versus the available similar sequences in the NCBI.

\section{Medium used for cellulase production}

The cultural medium used for the production of cellulase enzyme has the following constituents: Cellulose (Sigma) $(0.5 \mathrm{~g}), \mathrm{NaNO}_{2}$ $(0.2 \mathrm{~g}), \quad \mathrm{K}_{2} \mathrm{HPO}_{4}(0.1 \mathrm{~g}), \quad \mathrm{MgSO}_{4} .7 \mathrm{H}_{2} \mathrm{O} \quad(0.05 \mathrm{~g})$ and $\mathrm{KCl}(0.05 \mathrm{~g})$ up to $100 \mathrm{ml}$ distilled water and the $\mathrm{pH}$ was adjusted to 5.7 . Fractions of $50 \mathrm{ml}$ of the medium were poured into $250 \mathrm{ml}$ Erlenmeyer flasks and autoclaved for $15 \mathrm{~min}$ at $121^{\circ} \mathrm{C}$ and 1.5 bars. After sterilization, the flasks were inoculated with a fungal disc $(9 \mathrm{~mm}$ diameter) taken from the periphery of 7-day-old cultured fungi grown in Dox agar plates. The inoculated flasks were incubated at $30^{\circ} \mathrm{C}$ for 7 days, then, the culture broth was filtered and cell filtrates were kept in the fridge to be used as a source for crude cellulase enzyme solution. 


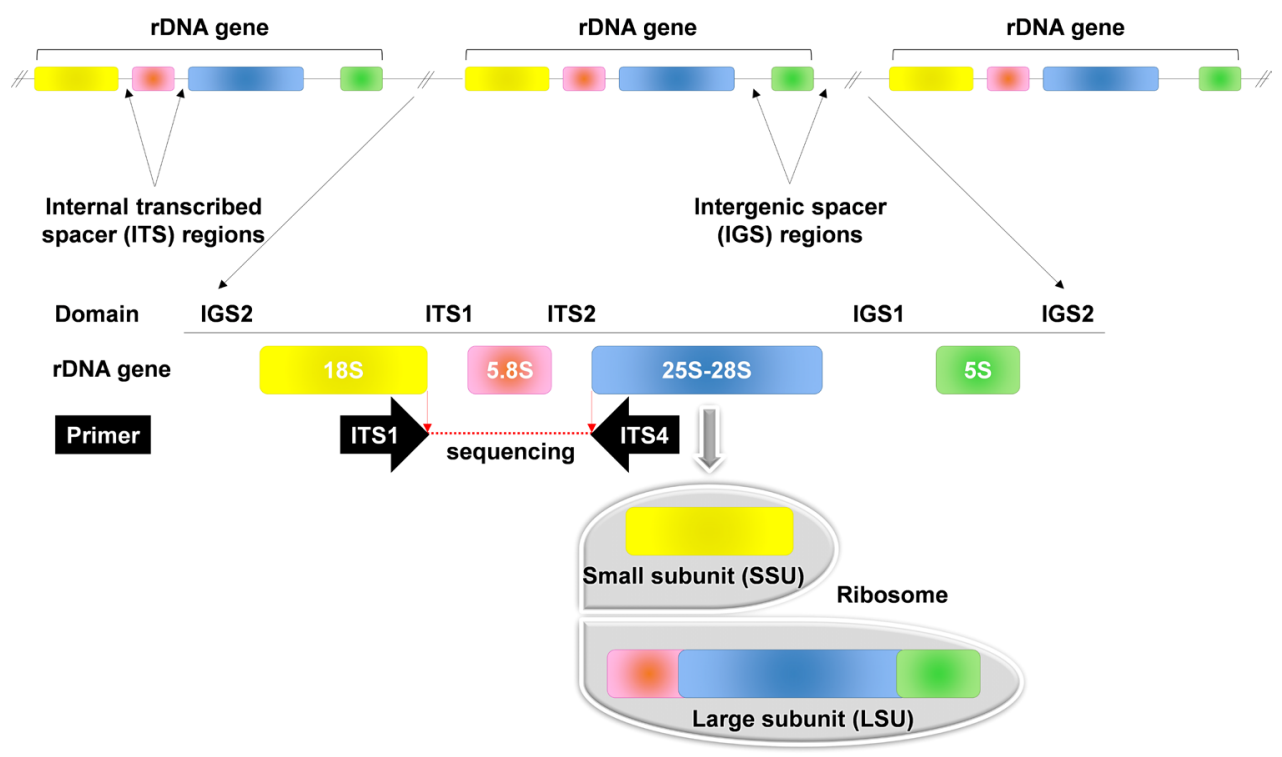

Fig. 1. Structure of the rDNA gene in fungi indicating the two regions that were sequenced using ITS1 and ITS4 primers for amplifying ITS (ITS1/5.8S/ITS2) region [The drawing was edited after Macrogen Inc. (Seoul 08511, Republic of South Korea)].

Screening for cellulose producing fungi

Filtrates were used for screening of cellulolytic activity on assay medium, which contained cellulose (Sigma) $(0.1 \%)$ and agar $(1.5 \%)$ in phosphate buffer $(0.05 \mathrm{M}, \mathrm{pH} 5.7)$ (Gomori, 1955), using cup clear zone technique (Ingram, 1951). The medium was sterilized as previously mentioned. The assay plates were prepared by pouring $20 \mathrm{ml}$ of the assay medium in each sterile Petri dish $(9 \mathrm{~cm})$ and then allowed to cool and solidify. Two cups were made per each plate by a sterile cork borer $(9 \mathrm{~mm}$ in diameter). Aliquots of $100 \mathrm{ul}$ of the crude enzyme preparation was introduced into each cup. The plates were left for $15 \mathrm{~min}$, then incubated at $37^{\circ} \mathrm{C}$ for $48 \mathrm{hr}$. At the end of incubation period, the plates were flooded with $10 \mathrm{ml}$ Lugol's iodine solution (Biotic Research Corporation). The diameters of the developing clear zones were measured and the means of two clear zones per plate were calculated and taken as a criterion for cellulose activity. Experiment was done in completely randomized design with three replicates and multiple comparisons were performed based on Duncan's New Multiple Range test (Duncan, 1955).

\section{$\underline{\text { Results }}$}

Fungal isolation and molecular identification for cellulytic fungi

In the present study, eight cellulytic fungal isolates were recovered from rotten peel of taro corm (Colocasia esculenta) as a source of cellulase. These fungi were carefully identified by morphological characteristics including color of the colony, growth and presence or absence of exudates. Some of the microscopic characteristics include hyphae and spore structures. These new isolates were shown to belong to Fusarium solani, F. proliferatum, F. fujikuroi, F. equiseti, Penicillium lanosum and $P$. flavigenum. These primarily identified isolates were further characterized at the molecular level based on the divergence of the ITS region of rDNA gene.

BLAST analysis involving the sequences of the ITS region in the eight isolates was conducted and multiple comparison has proven that these isolates are closely related to Fusarium (M1-M6 isolates) and Penicillium (M7-M8 isolates) genera. A cladogram tree was generated for the DNA sequences of the eight isolates as well as those of other fungal genera and species (Fig. 2). These isolates were distributed in six different clades of which three isolates of Fusarium. namely M2 (accession no. MH553281), M5 (accession no. MH553284) and M6 (accession no. MH553285) exist in one clade, while each of the other isolates exists in a separate clade. The M2 isolate was closely related to Fusarium solani strain D866 (accession no. MH266068.1, 
99\% identity), while M5 and the M6 isolates were closely related to Fusarium solani isolate KUSF301 (accession no. MF136401.1, 100\% identity) and Fusarium solani isolate AA2I4F2 (accession no. KX421443.1, 96\% identity). The M1 isolate (accession no. MH553280) was closely related to Fusarium proliferatum isolate LSNZI7 (accession no. MH277349.1, 97\% identity), while M3 (accession no. MH553282) and M4 (accession no. MH553283) were closely related to F. fujikuroi strain Fv1 (accession no. MH282573.1, 100\% identity) and $F$. equiseti strain D83 (accession no. KX878912.1, 99\% identity), respectively. The M7 isolate (accession no. MH553286) was closely related to Penicillium lanosum strain F-7 (accession no. MF077234.1, 100\% identity), while M8 isolate (accession no. MH553287) was closely related to Penicillium flavigenum strain
CML 2965 (accession no. KR261446.1, 100\% identity) (Figs. 2 and 3).

Multiple sequence alignment of the eight cellulase-producing fungi resulted in the generation of cladogram tree describing the phylogenetic relationships within the new isolates is shown in Fig. 3.

The generated tree has proven that the three Fusarium solani M2, M5 and M6 isolates are closely related, while the highest relationship was found between the two Penicillium spp. M7 and M8 isolates (Fig. 4). Interestingly, the latter two species are in close relationship with the three Fusarium solani as compared to the three other Fusarium species.

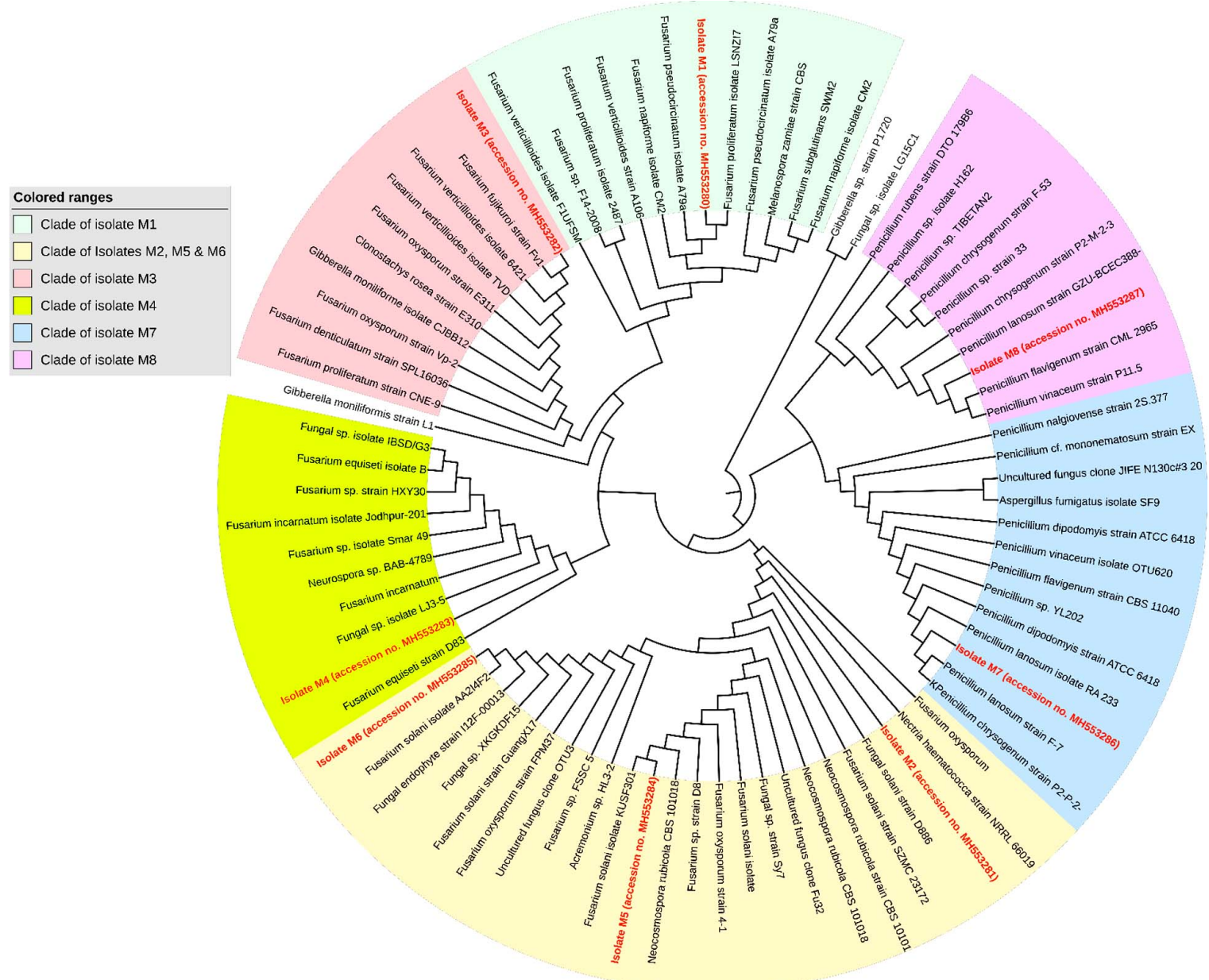

Fig. 2. Cladogram to describe phylogenetic relationships of the new isolates (M1-M8) with fungal genera whose sequences of ITS region of the rDNA gene are available in the NCBI [M1= Fusarium proliferatum, M2/ M5/M6= Fusarium solani, M3 = Fusarium fujikuroi, M4 = Fusarium equiseti, M7= Penicillium lanosum and M8= Penicillium flavigenum $]$. 
Screening for cellulase producing fungi on cellulose-agar plates

Screening for cellulase producing fungi on cellulose-agar plates revealed that the widest clear zone diameter was detected for M7 isolate, which belongs to $P$. lanosum followed by M8 and M1 isolates, which belong to P. flavigenum and $F$. proliferatum, respectively showing a mean clear zone diameters of $44.5,43.3$ and $41.3 \mathrm{~mm}$, respectively (Fig. 5).

M3 and M6 isolates, which belong to $F$. fujikuroi and F. solani, respectively, showed moderate clear zone diameters of 31.8 and $32.4 \mathrm{~mm}$, respectively. However, the other two F. solani M2 and M5 isolates as well as $F$. equiseti isolate M4 recorded the lowest clear zone diameters of 30,31 and $30 \mathrm{~mm}$, respectively (Fig. 5). It is well known that the widest the clear zone diameter, the highest the cellulase production by the fungus. As a model, two isolates with the highest (M7 isolate) and the lowest (M4 isolate) mean clear zone diameters are shown in Fig. 6.

$M 7 / 1-450$
$M 8 / 1-450$
$M 2 / 1-450$
$M S / 1-450$
$M 6 / 1-450$
$M 3 / 1-450$
$M 1 / 1-450$
$M 4 / 1-450$

Consensus

$M 7 / 1-450$ $M 8 / 1-450$ M2/1-450 MS/1-450 M6/1-450 M3/1-450 M1/1-450 M4/1-450

Consensus

$M 7 / 1-450$ $M 8 / 1-450$ M2/1-450 MS/1-450 $M 6 / 1-450$ M3/1-450 M1/1-450 M4/1-450

Consensus

$M 7 / 1-450$ M8/1-450 M2/1-450 MS/1-450 $M 6 / 1-450$ M3/1-450 M1/1-450 M4/1-450

Consensus

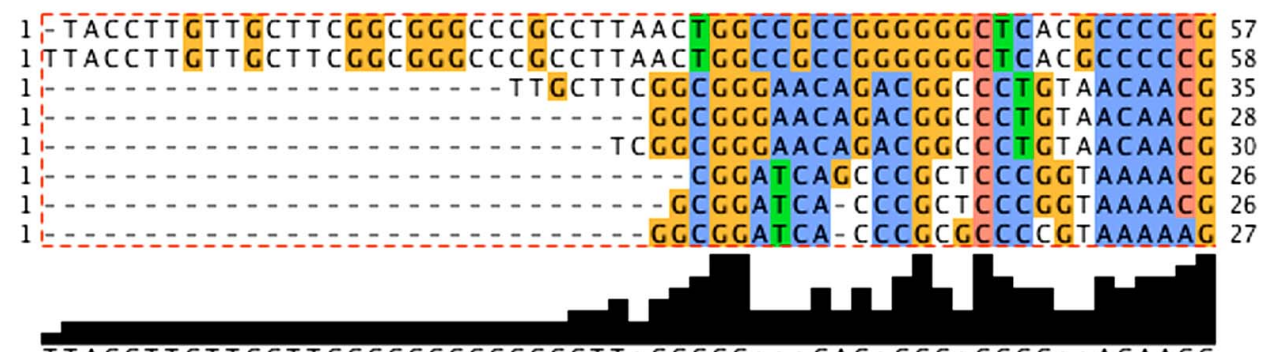

TTACCTTCTTCCTTCCCCCCCCCCCCCT T + CCCC +++CAC+CCC+CCCC++ACAACC

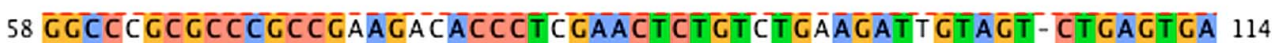
59 CCCCCCCCCCCCCCCAACACACCCTCCAACTCTCTCTCAACATTCTACT-CTCACTCA 115 36 CGCCGCC-CCCGCCACAGG-ACCCCTAA-CTCTCTTTTTATAAT CTTTTTCTCACTAA 90 29 GCCCGCC-CCCGCCAGAGC-ACCCCTAA-CTCTCTTTTTATAATCTTTTTCTCACTAA 83 31 GCCCGCC-CCCCCC-GAGG-ACCCCTAA-CTCTCTTTTTATAAACTTTTTCTGAGTAA 84 27 GCACGGC-CC-CCCACACG-ACCCCTAAACTCTGTTTCTATATCTAACTTCTCACTAA 81 27 GCACGCC-CC-GCCACACG-ACCCCTAAACTCTCTTTCTATATGTAACT TCTCACTAA 81 28 CCACGCC-CC-CCCCGACG-ACCCCTAAACTCTCTTTTTAT-IGCAACTTCTCACTAA 81

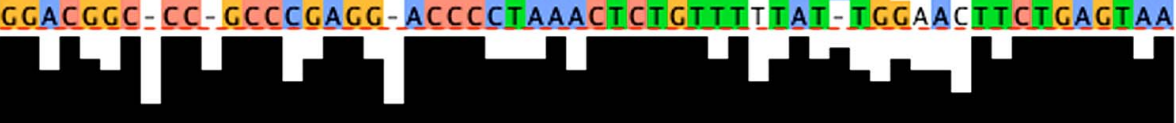

CGCCGCCCCCCCCCACACGCACCCCTAAACTCTCTTTTTATATTCTA+TTCTCACTAA

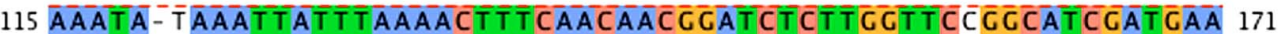
116 AAAT A-TAAAT TAT T TAAAACT T TCAACAACGCATCTCTTCGTTCCGGCATCCATCAA 172 91 A-CAACCAAATAAAT TAAAACT TTCAACAACGGATCTCTTCCCTCTGGCATCGATCAA 147 84 A-CAAGCAAATAAAT TAAAACT TTCAACAACGGATCTCTTGGCTCTGGCATCGATGAA 140 85 A-CAACCAAATAAAT TAAAACTTTCAACAACGCATCTCTTCGCTCTGCCATCGATGAA 141 82 AACCA - TAAATAAATCAAAACTTTCAACAAC G TATCTCTTCGTTCTGCCATCCATCAA 138 82 AACCA - TAAATAAATCAAAACTTTCAACAACGGATCTCTTGGTTCTGCCATCGATGAA 138 82 AACAAACAAATAAAT CAAAACTTTCAACAACGGATCTCTTCGTTCTGGCATCGATGAA 139 10 บ1

AACAAG+AAATAAATTAAAACTTTCAACAACCGATCTCTTGCTTCTCGCATCGATGAA

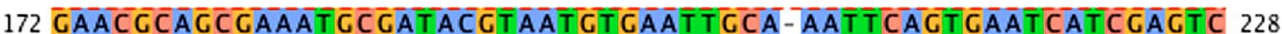
173 CAACGCAGCGAAAT GCCATACCTAAT GTCAAT TCCA-AATTCAGTCAATCATCCAGTC 229 148 GAAC GCA C CGAAAT GC GATAACTAAT GT GAAT T GCAGAAT T CAGT GAATCATCCAATC 205 141 GAACGCACCGAAAT GCGATAACTAAT GTCAAT T GCACAAT TCAGTCAATCATCCAATC 198 142 AAAC CCAACGAAAT GCCATAACTAAT CTCAAT TCCAAAAT TCAGTCAATCATCCAATC 199 139 GAACGCACCAAAAT GCCATAACTAAT CT GAAT TCCACAAT TCAGTCAATCATC CAATC 196 139 CAAC CCACCA GAAT CC GATAACTAAT CTCAAT TCACAAT T CACT CAATCATCCAATC 196 140 CAACCCAGCACAAT GCCATAAGTAAT GTCAAT TCCACAAT T CAGTCAATCATCCAATC 197

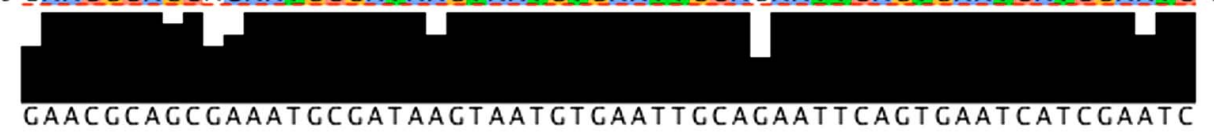

Fig. 3. Multiple sequence alignment for the eight new fungal isolates to generate cladogram tree describing phylogenetic relationships among them $[\mathrm{M} 1=$ Fusarium proliferatum, M2/M5/M6= Fusarium solani, M3= Fusarium fujikuroi, M4= Fusarium equiseti, M7= Penicillium lanosum and M8= Penicillium flavigenum]. 
$M 7 / 1-450$ M8/1-450 M2/1-450 MS/1-450 M6/1-450 M3/1-450 M1/1-450 M4/1-450

Consensus

M7/1-450 M8/1-450 M2/1-450 MS/1-450 M6/1-450 M3/1-450 M1/1-450 M4/1-450

Consensus

$M 7 / 1-450$ M8/1-450 M2/1-450 MS/1-450 M6/1-450 M3/1-450 M1/1-450 M4/1-450

Consensus

M7/1-450 M8/1-450 M2/1-450 MS/1-450 M6/1-450 M3/1-450 M1/1-450 M4/1-450

Consensus

M7/1-450 M8/1-450 M2/1-45O MS/1-450 M6/1-450 M3/1-450 M1/1-450 M4/1-450

Consensus

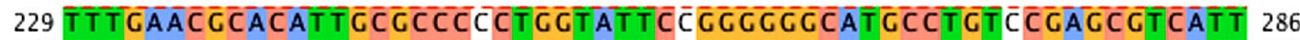

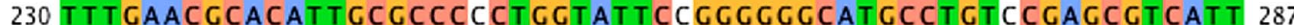
206 T TT CAACCCACAT TCCCCCCCCCACTAT TCT CCCCCCCATCCCTCTTCCACCCTCATT 263

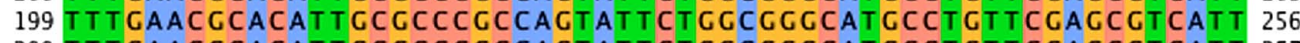

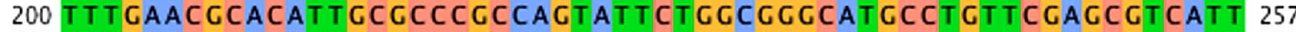
197 T T T GAACCCACAT TCCCCCCCCCACTAT TCT GGCGCGCATGCCTCTTCGACCGTCAT T 254 197 T T T GAACGCACAT T GC CCCCGCCACTAT TCT GGCGGGCATGCCTGT TCGACCGTCAT T 254

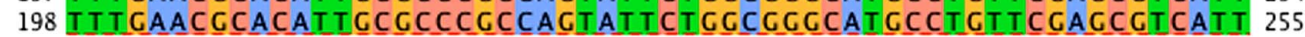

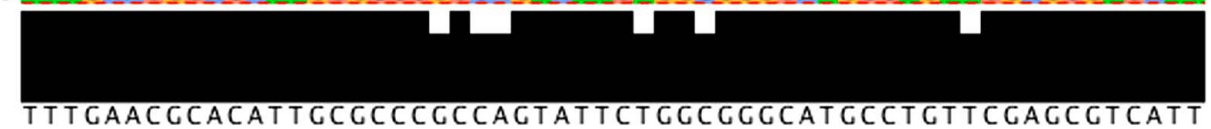

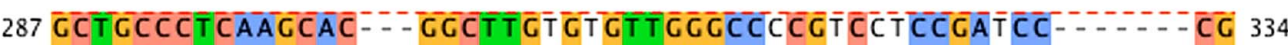
288 CCT GCCCTCAACCAC - - - GGCTTCTGTGTTCGGCCCCGTCCTCCGATCC - - - - - - CG 335 264 ACAACCCTCAGCCCCCCGGGCCTG-GCGTTGGGGATCGGCGGAACCCCCCTGTGGCCA 320 257 ACAACCCTCAGGCCCCCGGGCCTG-GCGTTGGGCATCGGCGGAACCCCCCTGTCGGCA 313 258 ACAACCCTCACGCCCCCGGCCCTC-GCGTTCGGCATCGCCGCAACCCCCCTGTGCCCA 314 255 TCAACCCTCAACCCC- - - AGCTTG-GTGTTGGGACTCG-CGACT- - - - - - - - CA 295

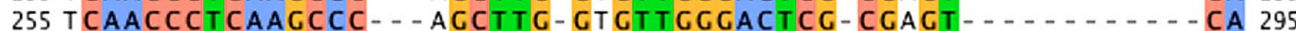
256 ICAACCCTCAACCTC = ACCTTC-CGCTTCGCACTCG-CGG-:-:

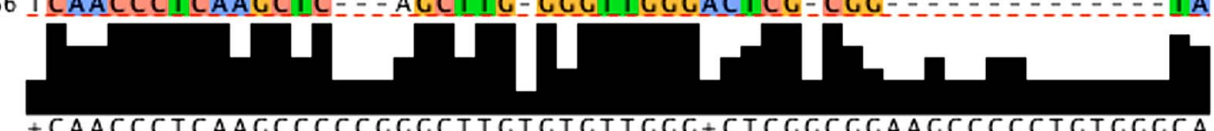

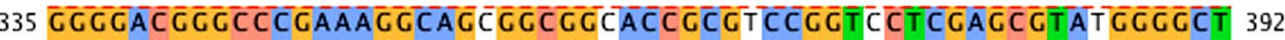
336 GGGCACGCGCCCGAAACCCAGCGCCGGCACCGCGT C CGGTCCT CGACCGTATGCGCCT 393 321 CACGCCGTCCCTCAAATACACTCGCGGTCCCGCCGCAGCTTCCATTGCGTACTAGCTA 378 314 CACGCCGTCCCTCAAATACAGT GGCGGTCCCGCCGCACCTTCCATTGCGTAGTAGCTA 371 315 CACCCCCTCCCTCAAATACACTCCCGCTCCCCCCCCACCTTCCATTCCCTATCTATCT 372 296 AAT CGCGTT CCCCAAAT T GATT GCCGGT CACGTCG-ACCTTCCATACCGTACTACTAA 352 296 AAT CGCCTTCCCCAAATT GATTCGCGGTCACCTCG-ACCTTCCATATCGTACTAATAA 352 295 ACCCCCCTICCCCAAATCGATICCCGCTCACCICC-ACCTICCATACCCTACTAAT CA 351

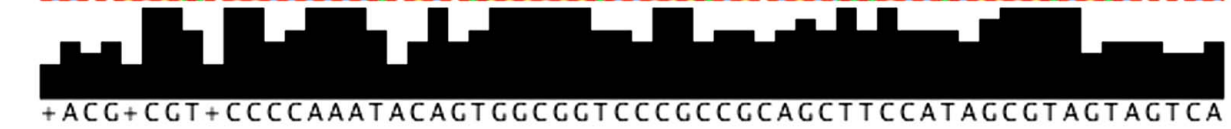

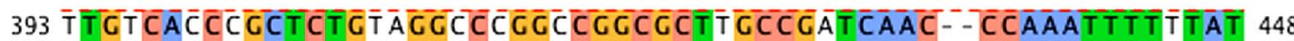

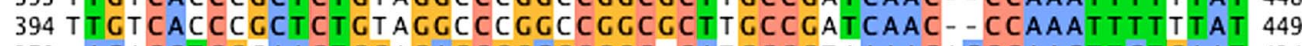
379 - ACACCTCGCAACTGGAGAGCGGCGCGGC-CATGCCGTAAAACACCCAACTTCTCAAT 434 372 - ACACCTCGCAACTGGACAGCGGCGCGGC-CATGCCGTAAAACACCCAACTTCTGAAT 427 373 AACTCCTCGCAATTGAGAGAGCGAGCGAC-GGC-CCACTCCCC-GTCAACAACCACAC 427 353 AAC-CCTCCTTACTGCTAATCCTCCCGCC-CACGCCCTTAAAC- CCCAACTTCTCAAT 407 353 AAC-CCTCCTTACT GATAATCATCACGGC-CACGCCCATAAAC-CCCAACTT CTGAAT 407 352 IACACCTCGTIACTGGTAATCGICGCGCC-CACGCCCTAAAAC-CCCAACTTCTCAAT 407

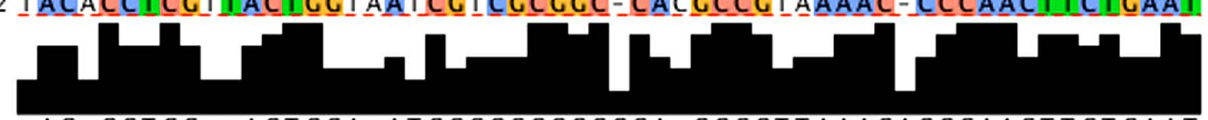

$+\mathrm{AC}+\mathrm{CCTCC}++\mathrm{ACTCCA}+\mathrm{ATCCCCCCCGCCCA+CCCCTTAAACACCCAACTTCTCAAT}$

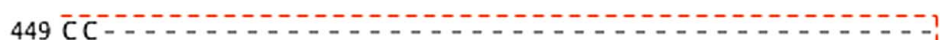

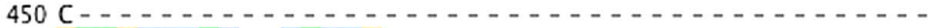

435 CTTCACCTCСAATCAC $\ldots \ldots \ldots \ldots$

428 СТ TСACСТССАAT САССТАCGAA $\ldots \ldots \ldots$

428 ACACAC- TCTACT CGT ACAT TCAT $\ldots \ldots \ldots$

408 CT TCACCTCGCATCAGCTAGCAATACCCGCTGAACT TAACCAT!

408 CT T GACCT C GCATCAC CTAT GAAT AC C C C C T GACT TAACCA

408 GT TCACCTCCGATCAGCTACCAATACCC C T CAACTTAACCAT

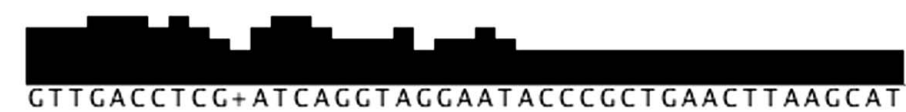

Fig. 3. Cont.

Discussion

Cellulose is the structural component of the primary cell wall of plants, many forms of algae and oomycetes (Chesson, 1987). Degradation of cellulosic materials is a complex process, which requires participation of microbial cellulolytic enzymes. Over years, a number of organisms, e.g., fungi and bacteria, that possess cellulose-degrading enzymes have been isolated and studied extensively (Budihal et al., 2016 and Ahmed et al., 2017). However, 
fungi are well known agents of decomposition of organic matter, in general and of cellulosic substrate in particular (Lynd et al., 2002). Cellulolytic enzymes play an important role in natural biodegradation processes in which plant lignocellulosic materials are efficiently degraded by cellulolytic fungi, bacteria, actinomycetes and protozoa (Wen et al., 2005). These enzymes also play a key role in cleaning the environment from harmful wastes, which are the main sources of pollution.

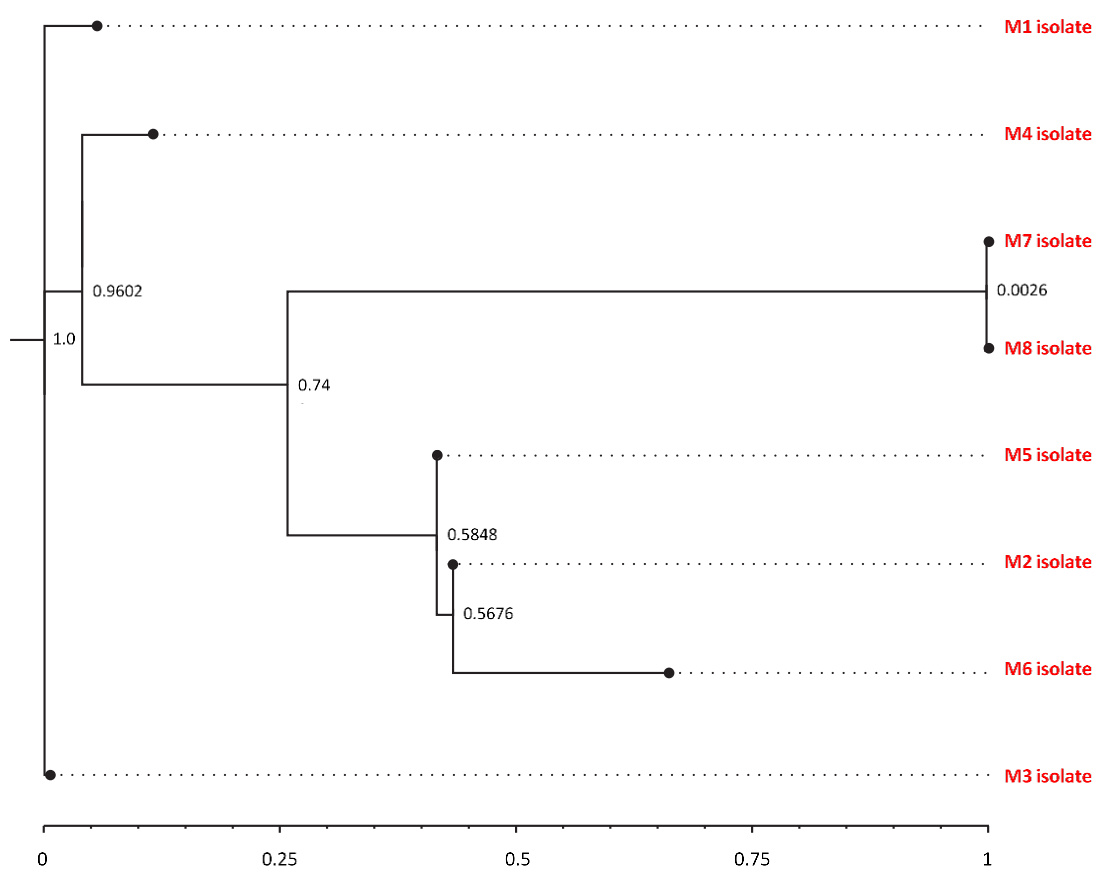

Fig. 4. Phylogenetic relationships among the fungal isolates (M1-M8) based on sequences of ITS region of the rDNA gene. Isolates inside red boxes belongs to Fusarium spp., while those in the blue box belong to Penicillium spp [Digital numbers on the scales represent the relationships between or among fungal species and genera].

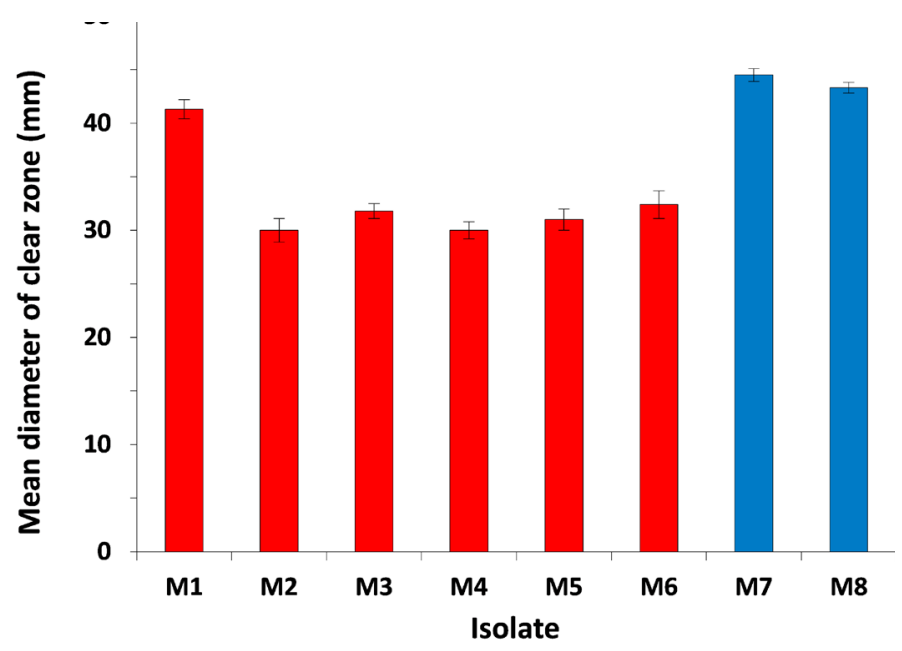

Fig. 5. Means of diameter of clear zone $(\mathrm{mm})$ to screen the new fungal isolates for their cellulase production on cellulose-agar plate after incubation for $48 \mathrm{hr}$ at $37^{\circ} \mathrm{C}$. Isolates represented in red columns (i.e., M1-M6) are closely related to Fusarium spp., while those represented in blue columns (i.e., M7 \& M8) are closely related to Penicillium spp. $[\mathrm{M1}=F$. proliferatum, M2/M5/M6 $=F$. solani, M3 $=F$. fujikuroi, M4 $=$ F. equiseti, M7= P. lanosum and M8=P. flavigenum $]$. 


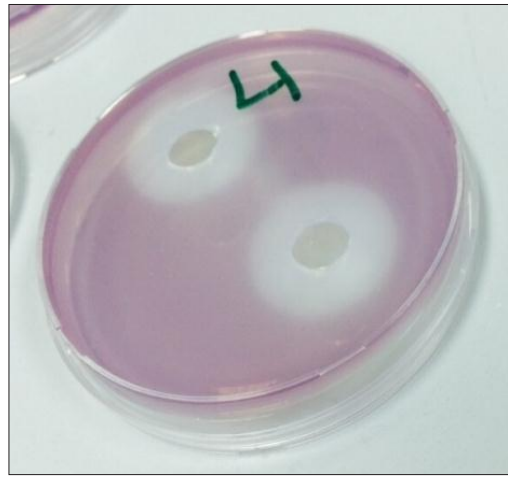

M4 isolate

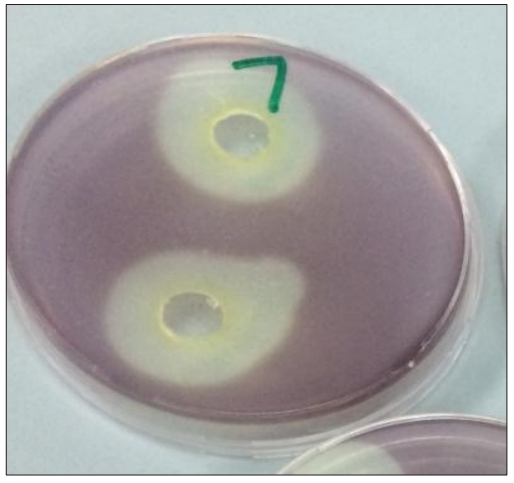

M7 isolate

Fig. 6. As a model, performance of two out of the eight fungal isolates in terms of clear zone reflecting the potential production of cellulase on cellulose-agar plates $[\mathrm{M} 4=$ Fusarium equiseti and M7 = Penicillium lanosum $]$.

In the present study, eight fungal species were isolated from rotten peel of taro corm (Colocasia esculenta). These fungi were screened for the potential production of cellulase enzyme where they gave clear zones ranging from 30.0$44.5 \mathrm{~mm}$. In this connection, a number of other cellulolytic fungi were previously isolated from Indian habitat (Ram et al., 2014 and Rathore et al., 2014). In Saudi Arabia, Bahkali \& Khiyami (1996) showed that thirty fungal species related to 15 genera collected from 30 soil samples were cellulase producing fungi. According to locations, the maximum number of fungal species was isolated from Dammam (20 species) followed by Niomas (18 species), Makkah and Riyadh (17 species each), Tabouk (16) species and Jizan (11 species). The dominant isolated fungal genera were Aspergillus, Pencillium, Alternaria, Ulocladium and Curvularia. Several investigations revealed that species of Trichoderma and Aspergillus are the main producers of cellulase followed by few species of bacteria and actinomycetes. Jahangeer et al. (2005) also isolated cellulolytic-fungi belonging to genera Trichoderma, Fusarium, Alternaria, Rhizopus, Aspergillus and Penicillium. The latter two genera showed highest potential for producing cellulose by Lakshmi \& Narasimha (2012). Plants used as substrates for cellulase production include sugarcane (Brijwani et al., 2010) and wheat bran (Sun et al., 2008). More recently, taro root was proven to be a suitable substrate for producing cellulase (Jadeja \& Verma, 2017). The latter plant was our target for isolating the different fungal isolates.

The concept of waste-to-energy is recently adopted towards the avoidance of pollutants and utilization of biomass encompasses in industry. Among plant-based biomass, lignocellulose is a potential substrate for renewable energy. Conversion of plant biomass avoids burning off and generation of air pollution. Fungi is almost responsible for $80 \%$ of the cellulose biodegradation on the globe (Moore Landecker, 1996). Fungi isolated in the present study belong to members of cellulose-decomposing fungi (Mehrotra \& Aneja, 1990). These new fungal isolates can be further utilized for commercial exploitation after being checked for thermostability $\left(40-70^{\circ} \mathrm{C}\right.$ and stability for $\left.2 \mathrm{hr}\right)$ that makes cellulose easily accessible for hydrolytic enzymes (Li et al., 2011). Fortunately, Penicillium, which is a potential producer of cellulase in the present study, has thermophilic versions that can grow well and decompose cellulose rapidly via action of thermostable cellulases (Picart et al., 2007).

It could be concluded that the ideal source for isolating cellulolytic fungi is the crude organic cellulose-containing material such as taro root. The fungal cultures isolated in the present investigation require further analysis to decipher their cellulolytic potential for converting cellulosic waste material into useful products to human and the environment.

\section{References}

Ahmed, A., Aslam, M., Ashraf, M., Al-Hassan, Nasim F., Batool, K. and Bibi, A. (2017) Microbial B-Glucosidases: Screening, characterization, cloning and applications. Journal of Applied \& Environmental Microbiology, 5, 57-73. 
Alexander, M. (1961) Microbiology of cellulose. In: "Introduction to Soil Microbiology" ( $2^{\text {nd }}$ ed.). John Wiley and Son Inc., New York and London.

Aro, N., Pakula, T. and Penttilä, M. (2005) Transcriptional regulation of plant cell wall degradation by filamentous fungi. FEMS Microbiol. Rev. 29, 719739 .

Bahkali, A.H. and Khiyami, M.A. (1996) Cellulosedecomposing fungi from Saudia Arabian soils. Qatar Univ. Sci. J. 16(1), 77-80.

Brijwani, K., Oberoi, H.S. and Vadlani, P.V. (2010) Production of a cellulolytic enzyme system in mixed-culture solid-state fermentation of soybean hulls supplemented with wheat bran. Process. Chem. 45, 120-128.

Budihal, S.R., Agsar, D. and Patil, S.R. (2016) Enhanced production and application of acidothermophilic Streptomyces cellulase. Bioresource Technology, 200, 706-712.

Chesson, A. (1987) Supplementary enzymes to improve the utilization of pigs and poultry diets. In: "Recent Advances in Animal Nutrition", Haresign, W., Cole, D.J.A. (Eds.), p71-89. London, Butterworths.

Duncan, D.B. (1955) Multiple range and multiple F-tests. Biom. 11, 1-42.

Ellis, M.B. (1971) Dematiaceous Hyphomycetes. Commonwealth Mycol. Inst. Kew. Surey England, p494.

Gilman, J.G. (1950) "Soil Fungi". The Iowa State College Press, USA.

Gomori, G. (1955) Preparation of buffers for use in enzyme active studies. Method in Enzymol. I, 138146.

Ingram, G.I.C. (1951) Formation of clear zones with 'sensitive' and 'resistant' Staphylococcus aureus in penicillin plate assays. Journal Genetics Microbiology, 5, 22-29.

Jadeja, G. and Verma, S. (2017) A Study on colocasia as a suitable substrate for production of cellulase. Int. J. Curr. Microbiol. App. Sci. 6, 2109-2119.

Jahangeer, S., Khan, N., Jahangeer, S., Sohail, M., Shahzad, S., Ahmad, A. and Khan, S.A. (2005)
Screening and characterization of fungal cellulases isolated from the native environmental source. Pak. J. Bot. 37, 739-748.

Lakshmi, A.S. and Narasimha, G. (2012) Production of cellulases by fungal cultures isolated from forest litter soil. Ann. For. Res. 55, 85-92.

Lederberg, J. (1992) Cellulases. In: "Encyclopaedia of Microbiology" (Vol. 1; A-C). Academic Press, Inc.

Letunic, I. and Bork, P. (2011) Interactive Tree Of Life v2: online annotation and display of phylogenetic trees made easy. Nucleic Acids Res, 39, W475-W478.

Li, D.C., Li, A.N. and Papageorgiou, A.C. (2011) Cellulases from thermophilic fungi: Recent insights and biotechnological potential. Enzyme Res. 1, 308730 .

Lynd, L.R., Weimer, P.J., van Zyl, W.H. and Pretorius, I.S. (2002) Microbial cellulose utilization: Fundamentals and biotechnology. Micro. Mol. Bio. Rev. 66, 506577.

Mehrotra, R.S. and Aneja, K.R. (1990) "An Introduction to Mycology", pp 141-143. $1^{\text {st }}$ ed. New Age (P) Ltd. Publishers, New Delhi.

Moore Landecker, E. (1996) "Fundamenttals of the Fungi". $4^{\text {th }}$ ed., New Jersey: Prentice Hall Inc.

Moubasher, A.H. (1993) "Soil fungi in Qatar and other Arab Countries". Centre for Scientific and Applied Research, University of Qatar, 566p.

Okonechnikov, K., Golosova, O., Fursov and MUGENE team (2012). Unipro UGENE: a unified bioinformatics toolkit. Bioinformatics, 28, 11661167.

Philippidis, G.P. (1994) Cellulase production technology. In: "Enzymatic Conversion of Biomass for Fuel Production", M. E. Himmel et al. (Eds.), ACS symposium series 566p.

Picart, P., Diaz, P. and Pastor, F.I. (2007) Cellulases from two Penicillium sp. strains isolated from subtropical forest soil: Production and characterization. Lett. Appl. Microbiol. 45, 108-113.

Ram, K., Kaur, K. and Sharma, S. (2014) Screening, isolation and characterization of cellulase producing micro-organisms from soil. Int. J. Pharm. Sci. Invent. 
3(3), 12-18.

Rathore, S.S. and Narendhirakannan, R.T. (2014) Screening of cellulase producing microorganisms from lake area containing water hyacinth for enzymatic hydrolysis of cellulose. Journal of Advanced Scientific Research, 5(3), 23-30.

Sajith, S., Priji, P., Sreedevi, S. and Benjamin, S. (2016) An overview on fungal cellulases with an industrial perspective. J. Nutr. Food Sci. 6, 1.

Seifert, K. (1996) Fuskey, Fusarium interactive Key. Agriculture and Agri-Food, Canada.

Singhania, R.R., Sukumaran, R.K., Patel, A.K., Larroche, C. and Pandey, A. (2010) Advancement and comparative profiles in the production technologies using solid-state and submerged fermentation for microbial cellulases. Enzyme Microb. Technol. 46, 541-549.

Sukumaran, R.K., Singhania, R.R. and Pandey, A. (2005) Microbial cellulases production, applications and challenges. J. Sci. Ind. Res. 64, 832.

Sun, X., Liu, Z., Qu, Y. and Li, X. (2008) The effects of wheat bran composition on the production of biomass-hydrolyzing enzymes by Penicillium decumbens. Appl. Biochem. Biotechnol. 146, 119128.
Vries, R.P. and Visser, R. (2001) Aspergillus enzymes involved in degradation of plant cell wall polysaccharides. J. Microbiol. Mol. Biol. 65, 497522.

Walsh G. (2002) Industrial enzymes: Proteases and carbohydrases. In: "Proteins, Biochemistry and Biotechnology", John Wiley and Sons. Ltd.

Walsh, G. (2015) Industrial enzymes: Proteases and carbohydrases. In: "Proteins. Biochemistry and Biotechnology", pp. 327-369. $2^{\text {nd }}$ ed. John Wiley and Sons, Inc.

Walter, H.R. (1998) Microcrystalline cellulose technology. In: "Polysaccharide Association Structure in Food". Marcel Dekker, Inc.

Wen, Z., Liao, W. and Chen, S. (2005) Production of cellulase by Trichoderma reesei from dairy manure. Bioresour. Technol. 96, 491-499.

White, T.J., Bruns, T., Lee, S. and Taylor, J. (1990) Amplification and direct sequencing of fungal ribosomal RNA genes for phylogenetics. In: "PCR Protocols: A Guide to Methods and Applications", Innis, N., Gelfand, D., Sninsky, J., White, T. (Eds.), pp. 315-322. Academic Press.

(Received 31/1/2019; accepted $23 / 3 / 2019)$

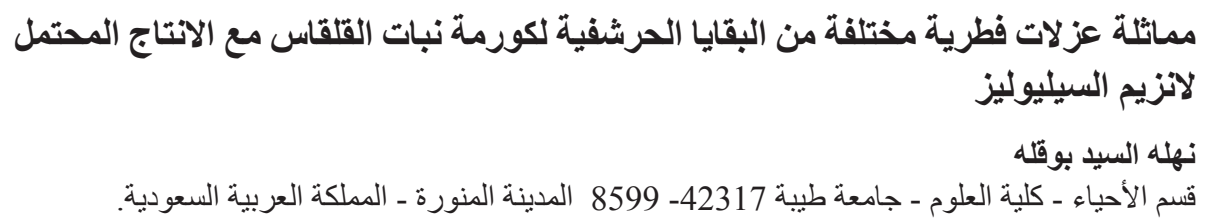

الهدف من العمل الحالي هو "عزل وتحديد“، الفطريات المختلفة من حطام الأوراق الحرشفية الخارجية البنية

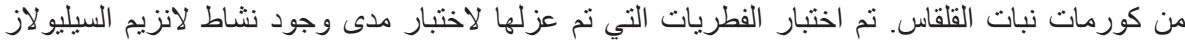

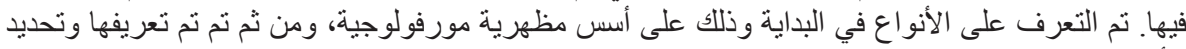

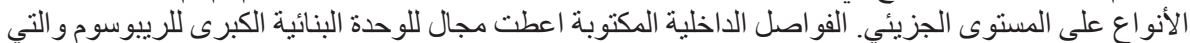

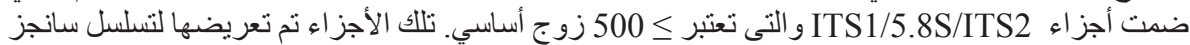

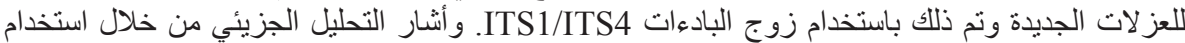

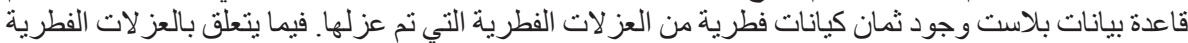

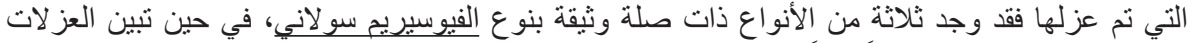

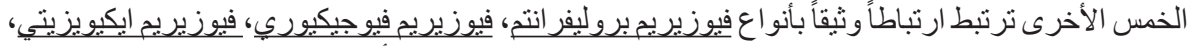

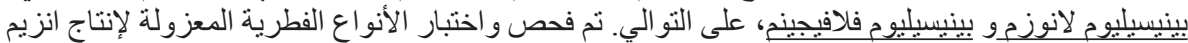

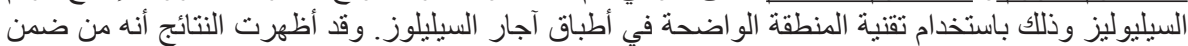

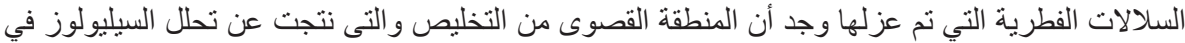

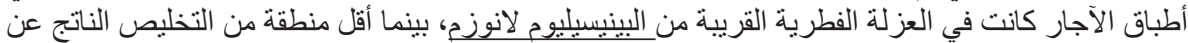

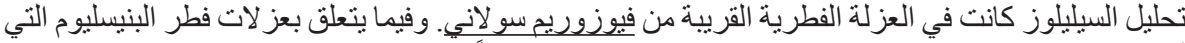

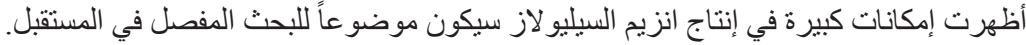

